\title{
CONSULTA MÉDICA SIMULADA COMO EXPERIENCIA DE APRENDIZAJE PARA ALUMNOS DEL PRIMER CICLO DE MEDICINA HUMANA DE LA UNIVERSIDAD NORBERT WIENER
}

\author{
SIMULATED MEDICAL CONSULTATION \\ AS LEARNING EXPERIENCE FOR STUDENTS \\ OF THE FIRST SEMESTER OF HUMAN MEDICINE \\ OF THE NORBERT WIENER UNIVERSITY
}

\author{
AGUSTÍN IZA STOLL \\ Universidad Norbert Wiener \\ PEDRO JESÚS MENDOZA-ARANA \\ Universidad Norbert Wiener \\ VÍCTOR CHOQUEHUANCA-VILCA \\ Universidad Norbert Wiener
}

\section{RESUMEN}

La simulación es empleada como un método cada vez más frecuente en la formación médica, tanto en la etapa clínica como en la preclínica. En este caso, nos interesó explorar su empleo en una vivencia profesional diseñada para alumnos del primer semestre de estudios, con el objetivo de identificar sus reacciones a una entrevista clínica simulada como desencadenante de reflexiones sobre el ser médico. En el primer ciclo de la Escuela de Medicina Humana, y como parte de un curso de profesionalismo médico, se programó a todos los estudiantes a una consulta médica ambulatoria simulada en la que podían jugar los roles de médico o paciente y posteriormente se les sometió a una encuesta. Esta población incluye a 18,7 \% de alumnos con una segunda carrera, siendo la primera de otras ciencias de la salud. La experiencia fue calificada como satisfactoria en el 90,6 \%; 97,4 \% respondieron que ayudó a consolidar su vocación, $99 \%$ catalogaron como importante el saludo al inicio de la consulta y $41 \%$ experimentaron alguna dificultad en el transcurso de la entrevista (nerviosismo, inseguridad). Se concluye que la simulación de una entrevista clínica es una experiencia educativa valiosa en alumnos del primer semestre de la carrera, y permite una amplia discusión sobre el ser médico en la carrera que se inicia.

Palabras clave: educación médica; recursos humanos en salud.

\section{ABSTRACT}

The simulation has been used as a method more and more frequent in the medical training both in the clinical stage and preclinical. In this case, we interested to explore its use in a professional experience design to first semester learners, whit the aim to identify their reactions in a simulated clinical interview such as trigger 
of reflections about being medico. In the first semester of Human Medical School, and as part of a subject of Medical Professionalism, all students were scheduled for a simulated outpatient medical visit, in this consult learners could play the roles of doctor or patient, after that they were subjected to a poll. This population includes to $18,7 \%$ of students of second career, being the first of other health sciences. The experience was graded as satisfactory in the 90,6\%; $97,4 \%$ answered that helped to consolidate their vocation, $99 \%$ indicated as important the greeting at the beginning of the medical appointment and $41 \%$ experienced some difficulties during of the interview (nervousness, insecurity). In conclusion the simulation of a clinical interview is an educational experience valuable in students of the first semester of the career and it permits a wide discussion about of being medico in the career that begins.

Key words: medical education, human resource in the health.

\section{INTRODUCCIÓN}

Uno de los elementos estructurales que se resiste a las innovaciones en diseño curricular en Medicina, es la diferencia entre el estadio preclínico y el clínico. La mayor parte de mallas curriculares de Medicina mantienen esta diferencia, y reservan el contacto con el paciente para las etapas avanzadas de la formación ${ }^{1}$. Sin embargo, la disponibilidad tecnológica y pedagógica de nuevas opciones, como la simulación, permite ahora brindar esta experiencia de aprendizaje de manera segura para el paciente $^{2}$; además, el impacto de tales experiencias sobre el proceso formativo puede ser muy significativo.

Por otro lado, hay una tendencia al desarrollo explícito de componentes tales como el profesionalismo, los cuales se deben trabajar a todo lo largo de la carrera, vale decir, desde el inicio o lo más temprano posible. Así mismo, la simulación viene siendo empleada como un método cada vez más frecuente en la formación médica, tanto en la etapa clínica como en la preclínica; en este caso, nos interesó explorar su empleo en una vivencia profesional diseñada para alumnos del primer semestre de estudios, con el objetivo de identificar las reaciones de alumnos de primer semestre a una entrevista clínica simulada, a efectos de desencadenar sus reflexiones sobre el ser médico.

\section{MATERIAL Y MÉTODOS}

Se trabajó en consultorios simulados, los mismos que cuentan con las dimensiones y equipamiento estándar para un consultorio médico, de acuerdo con la Norma Técnica de Salud vigente. Estos consultorios simulados cuentan con una sala de observación adjunta, separada del consultorio por un cristal Gessel.

Se concretó la experiencia en entrevistas clínicas simuladas, en las cuales un alumno efectuaba el rol de médico, y el rol de paciente fue asumido en la mayoría de los casos por el docente; en algunos casos por otro alumno. El aula Gessel permitía que aquellos alumnos que no participaban directamente en la entrevista la observaran, para luego evaluar y discutir en conjunto los diversos aspectos de la misma. Las indicaciones para esta consulta, debido a que los alumnos de primer ciclo no tienen 
todavía los conocimientos nosológicos, incluia recibir y saludar al paciente, preguntarle por filiación, antecedentes familiares y personales, $y$, finalmente, el motivo de la consulta.

Se dividió a los alumnos en grupos de 12; en los que dos de ellos efectuaban los roles ya descritos, y los restantes 10 observaban la experiencia. Luego se procedió a rotar a otros dos alumnos, generando cuatro experiencias por cada grupo, al cabo de las cuales se llevaba a cabo una discusión por un espacio de 30 minutos.

Previamente, todos los alumnos participaron en una sesión teórica de 45 minutos, brindada por uno de los autores, de tipo exposición dialogada y participativa, en la cual se presentaron y discuteron conceptos sobre la vocación médica -enfatizando la importancia de la relación humana entre médico y paciente- y se impartieron las indicaciones para la actividad práctica. Los tres autores cumplieron el rol de facilitadores de la experiencia, y de su deconstrucción posterior.

Una semana después de sucedida la experiencia, se aplicó una encuesta autoadministrada, consistente en 11 preguntas, 7 cerradas y 4 abiertas, que luego se ingresaron a una base de datos y fue analizada empleando el software Epi-Info versión 3.5.4.

\section{RESULTADOS}

\section{Edad de los estudiantes}

Se encuestó a todos los estudiantes de una misma cohorte de ingresantes. De los 191 estudiantes encuestados, el rango de edad va de 15 a 47 años, con una mediana de 17 años. La gran mayoría se encuentra entre los 16 y19 años: 131, lo cual constituye el $68 \%$ del total.

\section{Sexo}

El alumnado mayoritariamente es femenino, 130 de 196, lo que representa 2/3 del total, siendo la mediana de 17 ańos para ambos sexos.

\begin{tabular}{lcr}
\hline Sexo & Frecuencia & Porcentaje \\
\hline Masculino & 66 & $33,7 \%$ \\
Femenino & 130 & $66,3 \%$ \\
\hline Total & 196 & $100 \%$
\end{tabular}

\section{Ingreso a la universidad}

De acuerdo a las 193 respuestas, la gran mayoría postuló a Medicina como su primera carrera profesional: 157 (81,3 \%), mientras que para $18,7 \%$ esta era una segunda carrera, es decir, ya contaban con un título profesional.

\begin{tabular}{lcc}
\hline Carrera & Frecuencia & Porcentaje \\
\hline Primera & 157 & $81,3 \%$ \\
Segunda & 36 & $18,7 \%$ \\
\hline Total & 193 & $100 \%$
\end{tabular}

\section{Papel que representaron}

\begin{tabular}{lcc}
\hline Rol & Frecuencia & Porcentaje \\
\hline Médico & 91 & $46,9 \%$ \\
Paciente & 39 & $20,1 \%$ \\
Observador & 64 & $33,0 \%$ \\
\hline Total & 194 & $100 \%$
\end{tabular}

\section{Papel de preferencia}

La mayoría hubiera preferido el rol de médico 157 (83,1\%), en lugar de ser paciente u observador.

\begin{tabular}{lcc}
\hline Rol Preferido & Frecuencia & Porcentaje \\
\hline Médico & 157 & $83,1 \%$ \\
Paciente & 22 & $11,6 \%$ \\
Observador & 10 & $5,3 \%$ \\
\hline Total & 189 & $100 \%$
\end{tabular}




\section{Importancia del saludo}

\section{y la presentación}

El 99 \% (194 de 196) consideraron que el saludo y la presentación inicial del médico en la entrevista fue muy importante o importante.

\begin{tabular}{lcc}
\hline Saludo & Frecuencia & Porcentaje \\
\hline Muy importante & 158 & $80,6 \%$ \\
Importante & 36 & $18,4 \%$ \\
Poco importante & 1 & $0,5 \%$ \\
Irrelevante & 1 & $0,5 \%$ \\
\hline Total & 196 & $100 \%$
\end{tabular}

\section{Orientación para la entrevista}

De las 194 respuestas, 154 (79 4\%) indicaron que los alumnos se sintieron apropiadamente orientados para llevar a cabo la entrevista.

\begin{tabular}{lcc}
\hline Orientado & Frecuencia & Porcentaje \\
\hline Sí & 154 & $79,4 \%$ \\
No & 40 & $20,6 \%$ \\
\hline Total & 194 & $100 \%$
\end{tabular}

\section{Sentimiento que experimentó} el alumno durante su participación

En esta pregunta abierta se obtuvieron diversas respuestas que se muestran en el cuadro.

\begin{tabular}{lcc}
\hline Sentimiento & Frecuencia & Porcentaje \\
\hline Nerviosismo & 75 & $38,7 \%$ \\
Incertidumbre & 34 & $17,5 \%$ \\
Tranquilidad & 69 & $35,6 \%$ \\
Dominio de escena & 16 & $8,2 \%$ \\
\hline Total & 194 & $100 \%$
\end{tabular}

\section{Vivencia durante la entrevista}

En esta pregunta abierta se obtuvieron diversas respuestas que se muestran en el cuadro siguiente. Destacan, por un lado: nerviosismo y sentirse asustado con un $27 \%$, ningún problema con 30,9\% y la necesidad de aprender más con $23 \%$.

¿Qué vivencia Frecuencia Porcentaje experimentó?

\begin{tabular}{lcr}
\hline Asustado & 6 & $3,1 \%$ \\
Nerviosismo & 48 & $24,7 \%$ \\
Estar haciendo mal & 14 & $7,2 \%$ \\
Hacer preguntas adecuadas & 12 & $6,2 \%$ \\
Ninguna & 60 & $30,9 \%$ \\
Necesidad de aprender más & 45 & $23,2 \%$ \\
Buena explicación previa & 2 & $1,0 \%$ \\
Ser observador y no poder & 7 & $3,6 \%$ \\
\hline intervenir & 194 & $100 \%$
\end{tabular}

\section{Satisfacción respecto de la experiencia}

La experiencia fue considerada satisfactoria por el 90,6\% de los participantes.

\begin{tabular}{lcc}
\hline Satisfactoria & Frecuencia & Porcentaje \\
\hline Sí & 174 & $90,6 \%$ \\
No & 18 & $9,4 \%$ \\
\hline Total & 192 & $100 \%$
\end{tabular}

\section{Ayuda a consolidar la vocación} El 97,4 \% de un total de 196 alumnos considera que la experiencia fue positiva para consolidar o afirmar su vocación de médico.

\begin{tabular}{lcc}
\hline Vocación & Frecuencia & Porcentaje \\
\hline Sí & 191 & $97.4 \%$ \\
No & 5 & $2,6 \%$ \\
\hline Total & 196 & $100 \%$
\end{tabular}




\section{DISCUSIÓN}

La introducción temprana de experiencias de contacto médico-paciente es una de las tendencias actuales en educación médica ${ }^{3}$, sin embargo, las nociones sobre lo que significa el término temprano varían; para algunos, una experiencia temprana es aquella que se da antes del tercer año en un curriculum de cinco años ${ }^{4}$.

Esta primera experiencia de entrevista para el estudiante del primer ciclo ejerciendo el rol de paciente o del médico, les impone un desafío no programado en su esquema de formación académica, altamente valorada por los participantes como un elemento de confirmación de su vocación.

La población ingresante a la Escuela de Medicina Humana de la Universidad Norbert Wiener es bastante joven, con una mediana de edad de 17 años, y mayoritariamente de sexo femenino. Este cambio es muy evidente comparado con la tendencia habitual de hace varias décadas, en la que el sexo masculino prevalecía ampliamente, lo cual representa el cambio de mentalidad de nuestra sociedad.

Se consideró que era crítico involucrar a los estudiantes desde sus inicios en el respeto a los derechos del usuario, así como incorporar valores y principios básicos en su desenvolvimiento profesional, transparentados en la comunicación con el paciente 5 . La información básica previa a la entrevista enfatizó la presentación y el saludo como una acción clave en la primera relación con el paciente, lo cual fue asimilado muy positivamente porque, en la respuesta, de 196 alumnos, el 80,6 \% consideró estas acciones como muy importantes, y el 18,4\% la calificó de importante, lo cual hacae un total del $99 \%$.
En cuanto a estar orientados en la entrevista, de los 196 alumnos, el 79,4\% respondió afirmativamente, mientras que el 20,6 \% fue negativo. Consideramos que esto se podría explicar en que para algunos alumnos la orientación no fue suficiente para el tipo de experiencia, no tanto desde el punto de vista técnico o procedimental -en lo cual se enfatizó en la orientación-, sino desde el punto de vista humano, pues era manifiesto para los docentes que participaron, que se trataba de una experiencia emocionalmente muy intensa para los participantes.

Si bien hubo un grupo de profesionales de la salud, que, por lo mismo, ya habían vivido dichas situaciones, para la mayoría resultó un desafío difícil de enfrentar, lo que se tradujo en una sensación de incertidumbre y nerviosismo.

En cuanto a las vivencias que experimentaron durante la entrevista, se puede consolidar en 3 respuestas, unificando 1 y 2 (nerviosismo y asustado), que representa al grupo con mayor dificultad y alcanza el 27,8 \%; luego ,el 3, 4 y 6 con $36,6 \%$, que apunta a haber hecho consciente la necesidad de aprender más para no volver a experimentar tal sensación, y finalmente las respuestas codificadas como 5, 7 y 8 con el $35,5 \%$ que señala no tener mayor dificultad.

En los que ejercían el rol de médico, su propia experiencia previa los deslizaba imperceptiblemente a "ser" el médico tradicional, orientado al aspecto reparativo y básicamente asistencial. Por parte de los alumnos que ejercían el rol de usuarios o pacientes, resultaba más laborioso teatralizar este rol, aunque los que contaban con alguna experiencia previa -alumnos que cursan una segunda carrera- tenían más recursos para improvisar una consulta. 
Es importante verificar que el 90,6\% de 192 respuestas asintieron que fue una experiencia muy satisfactoria, frente a un $9,4 \%$ que respondieron negativamente y que previsiblemente recoge a aquellos estudiantes que no se sintieron bien orientados en esta actividad.

\section{CONCLUSIONES}

Se concluye que la simulación de una entrevista clínica es una experiencia educativa valiosa en alumnos del primer semestre de la carrera, y permite una amplia discusión sobre el ser médico en la carrera que se inicia ${ }^{6}$. La evaluación general de la experiencia fue satisfactoria y sentida como positiva en su formación. La vocación sobre la carrera de Medicina Humana se reafirma con la respuesta afirmativa del $97,4 \%$ de los 196 alumnos que respondieron la encuesta. En esta respuesta abierta lo más resaltante es el pedido de mantener y practicar este tipo de experiencias con mayor frecuencia y que el rol a desempeñar sea como médico para ganar experiencia.

\section{REFERENCIAS BIBLIOGRÁFICAS}

1. Gordon JA, Hayden EM, Ahmed RA, Pawlowski JB, Khoury KN, Oriol NE (2010). Early bedside care during preclinical medical education: can technology-enhanced patient simulation advance the Flexnerian ideal?. Acad Med. 2010 Feb;85(2):370-7. Doi: 10.1097/ACM.0b013e3181c88d7.

2. Lateef F. (2010). Simulation-based learning: Just like the real thing. Journal of Emergencies, Trauma and Shock, 3(4), 348-352. http://doi. org/10.4103/0974-2700.70743.
3. Passi V, Doug M, Peile E, Thistlethwaite J, Johnson N (2010). Developing medical professionalism in future doctors: a systematic review. International Journal of Medical Education. 2010; 1:19-29.

4. Shuid AN, Yaman MN, Kadir RAA, Hussain RI, Othman SN, Nawi AM, Ugusman A, Daud F, RA Manap, Mohamed IN. (2015) Effect of early clinical skills teaching on 3rd year medical students' learning: The student perspective. Journal of Taibah University Medical Sciences (2015) 10(1), 26-32.

5. Ramasamy R, Sathish Babu Murugaiyan, Rachel Shalini, Kuzhandai Velu Vengadapathy, Niranjan Gopal (2014) Communication skills for medical students: An overview. Journal of Contemporary Medical Education 2(2):134. Doi: 10.5455/ jcme.20140321110500.

6. Perales, Alberto; Mendoza, Alfonso y Sanchez, Elard. Vocación médica: necesidad de su estudio científico. An. Fac. med. [online]. 2013, vol.74, n.2 [citado 2017-09-04], pp. 133-138. Disponible en: <http://www.scielo.org. pe/scielo.php?script=sci_arttext $\&$ pi$\mathrm{d}=S 1025-55832013000200009 \& 1-$ $\mathrm{ng}=\mathrm{es} \& \mathrm{nrm}=\mathrm{iso}>$. ISSN 1025-5583. 